\title{
Nonlinear mechanism of tsunami wave generation by atmospheric disturbances
}

\author{
E. Pelinovsky ${ }^{1,2}$, T. Talipova ${ }^{1}$, A. Kurkin ${ }^{2}$, and C. Kharif ${ }^{3}$ \\ ${ }^{1}$ Laboratory of Hydrophysics and Nonlinear Acoustics, Institute of Applied Physics, Russian Academy of Science, Nizhny \\ Novgorod, Russia \\ ${ }^{2}$ Department of Applied Mathematics, Nizhny Novgorod State Technical University, Nizhny Novgorod, Russia \\ ${ }^{3}$ Ecole Supérieure de Mécanique, Université de la Méditerranée, Marseilles, France
}

Received: 24 July 2001 - Revised: 29 October 2001 - Accepted: 30 October 2001

\begin{abstract}
The problem of tsunami wave generation by variable meteo-conditions is discussed. The simplified linear and nonlinear shallow water models are derived, and their analytical solutions for a basin of constant depth are discussed. The shallow-water model describes well the properties of the generated tsunami waves for all regimes, except the resonance case. The nonlinear-dispersive model based on the forced Korteweg-de Vries equation is developed to describe the resonant mechanism of the tsunami wave generation by the atmospheric disturbances moving with near-critical speed (long wave speed). Some analytical solutions of the nonlinear dispersive model are obtained. They illustrate the different regimes of soliton generation and the focusing of frequency modulated wave packets.
\end{abstract}

\section{Introduction}

Tsunami waves may be generated by underwater earthquakes, submarine landslides, rockslides, volcano explosions and rapid anomalous changes in the atmospheric pressure over the sea (Murty, 1977; Pelinovsky, 1996). For a tsunami to arise, it is necessary that the water surface deviate from its equilibrium on a sufficiently large area. In these cases the shallow water theory or long wave theory is the good theoretical and numerical model to describe the properties of the tsunami waves. In many cases, the tsunami source moves with variable speed and direction. Such a situation is typical for meteorological tsunamis and this mechanism is investigated by Efimov et al. (1985) and Rabinovich (1993). Meteorological tsunamis have occurred in the Mediterranean Sea and in the Okhotsk Sea (Rabinovich and Monserrat, 1998). In particular, near the Balearic Islands, the estimated atmospheric wave speed of $29 \mathrm{~m} / \mathrm{s}$ is very close to the phase speed

Correspondence to: E. Pelinovsky

(enpeli@hydro.appl.sci-nnov.ru) of long waves of $31 \mathrm{~m} / \mathrm{s}$ (Rabinovich and Monserrat, 1998), so the resonance effects should be very important.

The given paper analyzes the resonance effects of tsunami generation by moving atmospheric disturbances which lead to the appearance of anomalous large waves. The paper is organized as following. The nonlinear and corresponding linear shallow-water theory of tsunami generation by the atmospheric disturbances is briefly discussed in Sect. 2. The resonance character of tsunami generation is investigated in Sect. 3. Detailed calculations are given for 1D case, where all analytical expressions can be obtained in the explicit form. The similarity of the considered problem to the problem of tsunami generation by moving landslides is mentioned here (Pelinovsky, 1996; Pelinovsky and Poplavsky, 1996; Tinti and Bortolucci, 2000a, b; Tinti et al., 2001). A nonlineardispersive model of the resonant generated tsunami waves is briefly reproduced in Sect. 4. This model is based on the famous forced Korteweg-de Vries equation derived first by Akylas (1984). The solitary wave generation and interaction with moving forcing is studied in Sect. 5 based on the results by Grimshaw et al. (1994). There is a lot of various scenaria of wave dynamics depending on the speed and sign of the atmospheric disturbance. It is shown that the maximal variation of wave amplitude in the process of interaction can reach two to three times. The effect of wave focusing of nonlinear dispersive wave packets is analyzed in Sect. 6 . Such an effect is well-known for wind-generated waves and leads to the generation of freak or rogue waves (Osborne et al., 2000; Kharif et al., 2001). The same process of wave focusing with formation of the large-amplitude waves is possible for tsunami waves. Obtained results are summarized in the conclusion. The main goal of this paper is to demonstrate the use of possible analytical tests that characterize the role of nonlinearity, dispersion and forcing in the processes of the tsunami generation by atmospheric disturbances; such solutions are important for interpretation of field data and results of the numerical simulations. 


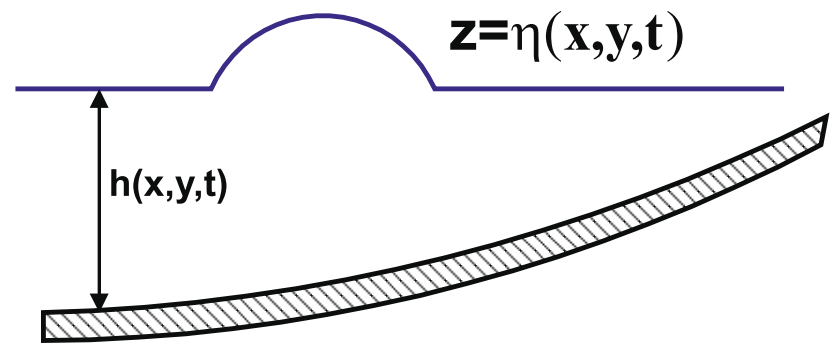

Fig. 1. Problem geometry.

\section{Shallow water model of the tsunami generation by at- mospheric disturbances}

The basic hydrodynamic model of tsunami generation by the atmospheric disturbances is based on the well-known Euler equations for ideal fluid on the non-rotated Earth

$$
\begin{aligned}
& \frac{\partial \boldsymbol{u}}{\partial t}+(\boldsymbol{u} \nabla) \boldsymbol{u}+w \frac{\partial \boldsymbol{u}}{\partial z}+\frac{1}{\rho} \nabla p=0, \\
& \frac{\partial w}{\partial t}+(\boldsymbol{u} \nabla) w+w \frac{\partial w}{\partial z}+\frac{1}{\rho} \frac{\partial p}{\partial z}=-g, \\
& \nabla \boldsymbol{u}+\frac{\partial w}{\partial z}=0
\end{aligned}
$$

with corresponding boundary conditions at the bottom and ocean surface (geometry of the problem is shown in Fig. 1). At the solid bottom $(z=-h(x, y))$,

$w-(\boldsymbol{u} \nabla) h=0$.

At the free surface $(z=\eta(x, y, t))$, the kinematic condition is

$w=\frac{d \eta}{d t}=\frac{\partial \eta}{\partial t}+(\boldsymbol{u} \nabla) \eta$,

and the dynamic condition,

$p=p_{a t m}(x, y, t)$.

Here $\eta(x, y, t)$ is the elevation of water surface, $\boldsymbol{u}$ and $w$ are horizontal $(\boldsymbol{u}=(u, v))$ and vertical components of the velocity field, $x$ and $y$ are coordinates in the horizontal plane; $z$-axis is directed upwards vertically, $\rho$ is water density, $p$ is pressure and $p_{a t m}$ is the variable atmospheric pressure, $g$ is gravity acceleration, $h(x, y)$ is the variable ocean depth. Differential operator, $\nabla$ acts in the horizontal plane $(\nabla=\{\partial / \partial x, \partial / \partial y\})$.

Usually tsunami waves are long (as compared with the ocean depth). Therefore, it is natural first to consider the long-wave (or shallow-water) approximation of the model of tsunami generation, and then to estimate conditions of its applicability. The theory of long waves is based on the main assumption that the vertical velocity and acceleration are low as compared to the horizontal ones and can be calculated from the initial system using the asymptotic procedure. As a small parameter it uses the ratio of the vertical velocity to the horizontal one or the ocean depth to the characteristic wavelength. Here a simpler algorithm is used, which consists of neglecting vertical acceleration, $d w / d t$ in Eq. (2). In this case Eq. (2) is integrated and, with the dynamic boundary condition (Eq. 6) taken into account, determines the hydrostatic pressure

$p=p_{a t m}+\rho g(\eta-z)$.

Substituting Eq. (7) into Eq. (1) and neglecting the vertical velocity once again, we obtain the first equation of the longwave theory

$\frac{\partial \eta}{\partial t}+(\boldsymbol{u} \nabla) \boldsymbol{u}+g \nabla \eta=-\frac{\nabla p_{a t m}}{\rho}$.

The second equation is yielded by integration of Eq. (3) over the depth from the bottom $(z=h(x, y))$ to the surface $(z=\eta(x, y, t))$, taking into account boundary conditions (Eq. 4 and 5), as well as the fact that horizontal velocity does not depend on vertical coordinate, $z$

$\frac{\partial \eta}{\partial t}+\nabla[(h+\eta) \boldsymbol{u}]=0$.

Equations (8 and 9) are closed as related to functions $\eta$ and $\boldsymbol{u}$. They are nonlinear (the so-called nonlinear shallowwater theory), inhomogeneous (the right-hand part is nonzero), and with variable coefficients due to $h(x, y)$.

The linear version of the shallow-water theory is most generally used within the tsunami problem. In this case variations of water depth are assumed weak, as well as the velocity of the fluid. As a result, we obtain a linear set of equations

$\frac{\partial \boldsymbol{u}}{\partial t}+g \nabla \eta=-\frac{\nabla p_{a t m}}{\rho}$,

$\frac{\partial \eta}{\partial t}+\nabla[h \boldsymbol{u}]=0$.

It is convenient to exclude $\boldsymbol{u}$ and pass over to the wave equation for the surface elevation

$\frac{\partial^{2} \eta}{\partial t^{2}}-\nabla\left(c^{2} \nabla \eta\right)=\nabla\left(\frac{h}{\rho} \nabla p_{a t m}\right)$,

where

$c(x, y)=\sqrt{g h(x, y)}$

is the speed of long wave propagation. Equation (12) is the basic one within the linear theory of tsunami generation and must be supplemented by the initial conditions. It is natural to believe that at the initial moment the ocean is quiet, i.e.

$\eta=0, \quad u=0, v=0, \quad$ or $\quad \partial \eta / \partial t=0$,

although due to linearity of Eq. (12), a more general case can also be considered. From the point of view of mathematical physics, the wave (Eq. 12) is too well studied to discuss the details of its solution here. The governing and long-wave systems presented here are the basic hydrodynamic models of tsunami generation by the atmospheric disturbances. 


\section{Long wave generation on a sea of constant depth}

The simplest model here uses the linear wave Eq. (12) with constant depth. In this case Eq. (12) reduces to the classical wave equation

$\frac{\partial^{2} \eta}{\partial t^{2}}-c^{2} \nabla^{2} \eta=\frac{h}{\rho} \nabla^{2} p_{a t m}$,

with constant long-wave speed, $c$ and zeroth initial conditions. Having replaced variable

$\zeta_{a}=\frac{h p_{a t m}}{\rho c^{2}}, \quad \eta=\zeta-\zeta_{a}$

Eq. (15) reduces to

$\frac{\partial^{2} \zeta}{\partial t^{2}}-c^{2} \nabla^{2} \zeta=\frac{\partial^{2} \zeta_{a}}{\partial t^{2}}$

In these variables the problem of tsunami wave generation by atmospheric perturbations is fully reduced to the analogous problem in the case of tsunami excitation by bottom displacements. Therefore, all the results of the linear shallow-water theory for "earthquake" tsunamis remain valid in this problem too. Note some differences, though. First, in the case of a static atmospheric perturbation, the solution of Eq. (17) becomes trivial, and the water level is equal to

$\eta=-\zeta_{a}=-\frac{h p_{a t m}}{\rho c^{2}}$

This relation is called "the law of the inverse barometer". Certainly, Eq. (18) can be supplemented with the constant which characterizes the average sea level under the average atmospheric pressure. Static "tracing" of atmospheric perturbations is typical for "atmospheric" problems and permits one to judge the values of atmospheric perturbations based on the data about changes in the sea level obtained, for example, from space.

Second, the piston model that is most characteristic for tsunami waves of seismic origin is not the main one here; it corresponds to the edge of the atmospheric disturbance, for example, to the difference in pressures on one side of the typhoon. And if we consider the atmospheric perturbation to be time-localized, then it corresponds to the bottom displacement that comes back to the initial state; by this, naturally, the amplitude of the excited waves will be smaller than that at the piston displacement. Thus, keeping other conditions equal, the meteorological tsunami is the strongest when a region of lower (higher) pressure (cyclone or anticyclone region) is formed quickly. If this region is stationary, or moving slowly, we have the complete analogy with the corresponding results for the piston model and there is no possibility to consider separately the generalization of the linear long-wave model. The Green function for waves generated by the atmospheric disturbances in the framework of linear potential theory is given by Kajiura (1963). However, if a cyclone is moving, a resonance between the cyclone velocity and the speed of tsunami waves is possible.
Let us consider the simplest one-dimensional model of cyclone when the atmospheric pressure is varied along the axis $x$ only, and a cyclone moves with constant speed $V$. The solution of Eq. (15), which satisfies the zeroth initial condition, is easily found explicitly (see for comparison, Pelinovsky, 1996; Tinti et al., 2001)

$$
\begin{gathered}
\eta(x, t)=\frac{c^{2}}{V^{2}-c^{2}} \zeta_{a}(x-V t)-\frac{c}{2(V+c)} \zeta_{a}(x-c t) \\
+\frac{c}{2(V+c)} \zeta_{a}(x+c t) .
\end{gathered}
$$

This solution is a superposition of three waves: one of them is bounded, and the two others are free. After some time they become separated in space: the first wave propagates together with the cyclone, and the other two leave it. Let us discuss first the field in the source for sufficiently long times, when the waves become separated in space; it is described by the first term in Eq. (19). We see that in the case of large speed $(V \rightarrow \infty)$ the surface elevation is very weak $\left(\eta \approx c^{2} \zeta_{a} / V^{2}\right.$ ), and when the cyclone motion is slow $(V \rightarrow 0)$ the surface level is the almost static disturbance Eq. (18). Of special interest is the case of synchronism between the cyclone motion and the excited wave, when even a small atmospheric disturbance causes strong water elevation (formally, infinite within this model). The free wave moving in the same direction with the cyclone is similarly amplified (but it is a trough, not a crest). The wave propagated in the opposite direction from the cyclone is limited with respect to the amplitude at any speed (that is caused by a great difference in velocities) and this wave is not resonant.

Similar calculations can also be made for the twodimensional atmospheric disturbances. Detailed calculations in the framework of Eq. (17) (formally, they were done for moving bottom displacements) were performed by Novikova and Ostrovsky (1978), and later confirmed within numerical modelling (Marchuk et al., 1983; Nosov and Shelkovnikov, 1995). It should be emphasized that the resonance is retained in the plane problems, wherein all the disturbances with velocities higher than $c$ are of a resonance character, and maximum radiation occurs along directions $\theta=\arccos (c / V)$, determined through the so-called Mach (Cherenkov) angle. Such relations are well-known in the theory of wave radiation. Wave amplitude stays finite at $c \neq V$, and it is proportional to the factor $\left(1-c^{2} / V^{2}\right)^{-1 / 2}$ for the Mach direction.

To estimate the accuracy of the linear model, let us consider the problem of tsunami generation by a moving cyclone within the nonlinear formulation. We will limit ourselves to the unidimensional variant of the nonlinear shallow-water theory (Eq. 8 and 9),

$$
\begin{aligned}
& \frac{\partial u}{\partial t}+u \frac{\partial u}{\partial x}+g \frac{\partial \eta}{\partial x}=-\frac{1}{\rho} \frac{\partial p_{a t m}}{\partial x}, \\
& \frac{\partial \eta}{\partial t}+\frac{\partial}{\partial x}[(h+\eta) u]=0 .
\end{aligned}
$$

Assuming that the elevation is only a function of the running coordinate $\zeta=x-V t$, we will try to find solutions for 


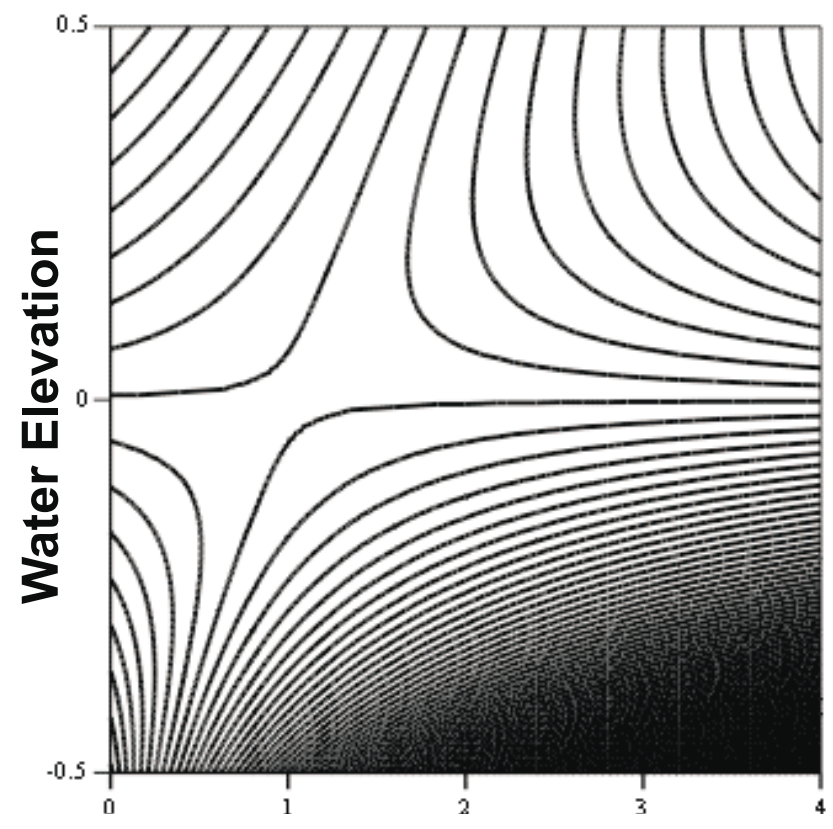

Froude Number

Fig. 2. Resonance curve for nonlinear generation of tsunami waves by atmospheric disturbance.

this system in the same form, i.e. depending on the same coordinate $\zeta$. Then the system (Eq. 20 and 21) yields nonlinear algebraic equations,

$(h+\eta) u=V \eta, \quad g \eta=V u-\frac{u^{2}}{2}-\frac{p_{a t m}}{\rho}$,

where it is assumed that there are no perturbations of the sea level and velocity outside the cyclone region. If bottom displacement is small and $V \neq c$, this algebraic system yields a linear relation (the same as the first term in Eq. 19). However, there is no unlimited growth of the amplitude under resonance $V=c$, and the corresponding resonance curve, "water elevation - Froude number", can be obtained from Eq. (22)

$p=\frac{2(F r-1) \zeta+(F r-4) \zeta^{2}-2 \zeta^{3}}{2(1+\zeta)^{2}}$,

where the Froude number $F r=V^{2} / g h$, and the water elevation is normalized on the basin depth, $\zeta=\eta / h$, and $P=p_{a t m} / \rho c^{2}$. This solution for different values of the atmospheric pressure $P$, is presented in Fig. 2. The curves are non-symmetric about the value $F r=1$, which is normal for nonlinear problems.

\section{Nonlinear dispersion model of resonant generated waves}

The formulae presented above yield out of the resonance $V=c$ that the wave field is well described within the lin- ear shallow-water theory. In the case of the resonance the effects of nonlinearity and dispersion are very important. Let us consider here a simplified model for resonance nonlinear generation of tsunami waves by the atmospheric disturbances including dispersion (Akylas, 1984; Pelinovsky and Choi, 1993). The equations of the nonlinear theory of long waves have been derived in Sect. 2. The dispersion effects are characterized by the vertical acceleration, $d w / d t$ in Eq. (2) which is ignored usually in long-wave theories. In the case of weak nonlinearity and dispersion, it can be calculated asymptotically (see for details, Pelinovsky and Choi, 1993). As a result, the following system can be derived for waves in the basin of constant depth

$$
\begin{aligned}
& \frac{\partial \boldsymbol{u}}{\partial t}+(\boldsymbol{u} \nabla) \boldsymbol{u}+g \nabla \eta=\frac{h^{2}}{3} \frac{\partial}{\partial t} \nabla^{2} \boldsymbol{u}-\frac{\nabla p_{a t m}}{\rho}, \\
& \frac{\partial \eta}{\partial t}+\nabla[(h+\eta) \boldsymbol{u}]=0 .
\end{aligned}
$$

These simplified equations (they are of the Boussinesq type) differ from the shallow-water Eqs. (8 and 9) in the presence of the high derivative of the horizontal velocity field in Eq. (24). This system provides the possibility of observing the process of tsunami generation by moving cyclone formations. Bearing in mind that the greatest efficiency of excitation is achieved in the resonant case, when the cyclone moves with the velocity, $V$ close to the linear long wave speed, $c$, we can further simplify the problem by considering it as a unidimensional one. This results in the following system,

$\frac{\partial u}{\partial t}+u \frac{\partial u}{\partial x}+g \frac{\partial \eta}{\partial x}=\frac{h^{3}}{3} \frac{\partial^{3} u}{\partial t \partial x^{2}}-\frac{1}{\rho} \frac{\partial p_{a t m}}{\partial x}$,

$\frac{\partial \eta}{\partial t}+\frac{\partial}{\partial x}[(h+\eta) u]=0$.

Taking into account the resonant character of tsunami generation, this system can be simplified. Let us re-write Eqs. (26 and 27) in the form of a nonlinear wave equation for the surface elevation $\eta$,

$\frac{\partial^{2} \eta}{\partial t^{2}}-c^{2} \frac{\partial^{2} \eta}{\partial x^{2}}=\prod\{\eta, u\}+\frac{h}{\rho} \frac{\partial^{2} p_{a t m}}{\partial x^{2}}$,

$\prod=-\frac{\partial}{\partial x}\left(\eta \frac{\partial u}{\partial t}\right)+\frac{h}{2} \frac{\partial^{2} u^{2}}{\partial x^{2}}-\frac{h^{3}}{3} \frac{\partial^{4} u}{\partial t \partial x^{3}}$.

Here, the right-hand part of Eq. (28) can be treated as proportional to a small parameter, characterizing the weak nonlinearity, dispersion and forcing. Using the linear relation, $u=\eta g / c$ in Eq. (29), Eq. (28) can be reduced to (Akylas, 1984)

$\frac{\partial \eta}{\partial t}+c \frac{\partial \eta}{\partial x}+\alpha \eta \frac{\partial \eta}{\partial x}+\beta \frac{\partial^{3} \eta}{\partial x^{3}}=\frac{\partial f}{\partial x}$,

where

$\alpha=3 c / 2 h, \quad \beta=c h^{2} / 6, \quad f=-h p_{a t m} / 2 c \rho$. 
Equation (30) is the forced version of the famous Korteweg-de Vries equation and currently it is considered as one of the basic equations of the nonlinear mathematical physics. Its solution contains the set of solitary waves (solitons) and dispersive tails (variable wave packets).

\section{Soliton generation and interaction with a moving forcing}

The numerical solutions of the forced Korteweg-de Vries equation described the wave generation by the moving atmospheric disturbance are given in the paper by Akylas (1984) and the following papers cited by Grimshaw et al. (1994). To demonstrate the roles of solitons in the forced dynamics of the water waves analytically, let us assume that the soliton has already been formed and consider the process of its amplification under the effect of a moving force. We suppose that the forcing is sufficiently weak, so that the soliton retains its form in the process of interaction (the condition for adiabatic interaction is slowness of variation of soliton parameters on distances of the order of the nonlinearity length). The solution for the forced Korteweg-de Vries equation is in the form of the asymptotic series

$$
\begin{aligned}
\eta(x, t) & =a(t) \operatorname{sech}^{2}(\Gamma(t)[x-V t-\Psi(t)])+\ldots, \\
\Gamma & =(\alpha a / 12 \beta)^{1 / 2}, \quad \Psi(t)=\int\left(p_{0}+\ldots\right) d t .
\end{aligned}
$$

The procedure for obtaining the equations for the soliton amplitude and velocity in the framework of the asymptotic method is well-known (see, for instance, Grimshaw et al., 1994). As a result, in the first approximation we obtain the energy balance equation for the amplitude,

$$
\frac{d}{d t} \int_{-\infty}^{\infty} \frac{\eta^{2}}{2} d x=\int_{-\infty}^{\infty} \eta \frac{\partial f}{\partial x} d x
$$

and for the position (phase) of the soliton we have the following unperturbed relation,

$\frac{d \Psi}{d t}=c-V+\frac{\alpha a}{3}$.

These equations describe the simplified model of the process of adiabatic interaction of the soliton with the moving force. Given a cyclone of short duration, 1 acts as the delta function, $f(x)=b \zeta(x / l)$ in Eq. (33), where $b$ is its intensity. For this function it is possible to calculate the integral in Eq. (33). As a result, the Eq. (33) has the differential form,

$\frac{d a}{d t}=-\frac{\alpha a b l}{12 \beta} \operatorname{sech}^{2} \Gamma \Psi \tanh \Gamma \Psi$.

After transformation, a $\rightarrow \Gamma, \Psi \rightarrow \theta=\Gamma \Psi$, the system (Eqs. 34 and 35) reduces to

$\frac{d \Gamma}{d t}=-\frac{\alpha b l \Gamma}{12 \beta} \operatorname{sech}^{2} \theta \tanh \theta$,

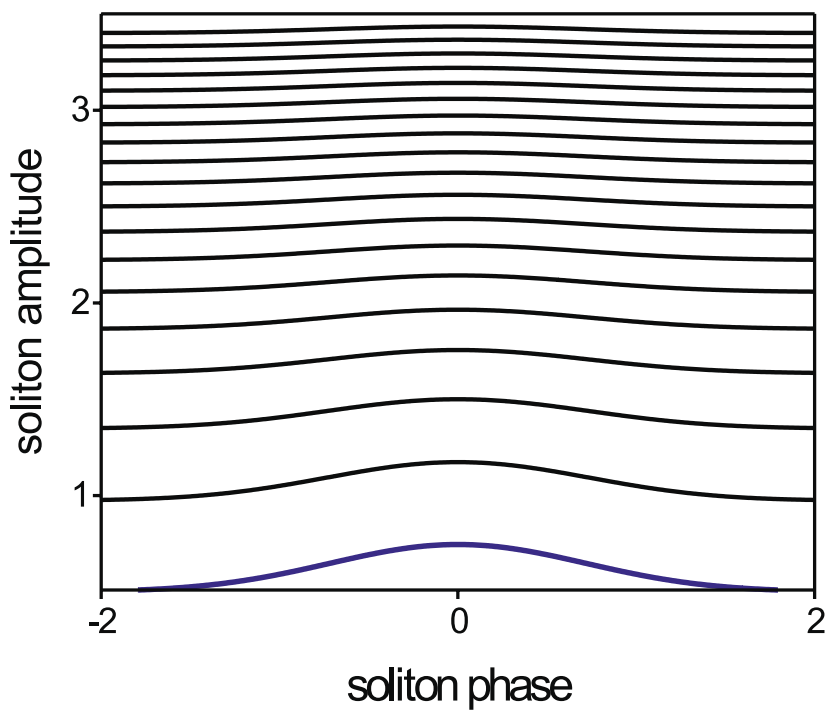

Fig. 3. Phase plane of system (Eqs. 36 and 37) for the "slow" moved atmospheric disturbance.

$\frac{d \theta}{d t}=\Gamma\left(c-V+4 \beta \Gamma^{2}\right)$.

All integral trajectories of this system can be found in the explicit form,

$\frac{\alpha b l}{24 \beta} \operatorname{sech}^{2} \theta=(c-V) \Gamma+\frac{4 \beta}{3} \Gamma^{3}+$ const.

After substitutions,

$$
\begin{gathered}
\alpha a_{0}=3|c-V|=12 \beta \Gamma_{0}^{2}, \quad G=\Gamma / \Gamma_{0}, \\
Q=\frac{\alpha b l}{24 \beta \Gamma_{0}|c-V|},
\end{gathered}
$$

(as it can be clearly seen below, $a_{0}$ is the amplitude of steady state generated soliton) the expression (Eq. 38) can be written in the dimensionless form,

$Q \operatorname{sech}^{2} \theta=-G \operatorname{sign}(V-c)+\frac{G^{3}}{3}+$ const.

The phase pattern of system (Eqs. 36 and 37), all the trajectories of which are determined by Eq. (40), depends on the signs of $(c-V)$ and $Q$. The last parameter in the physical variables is

$Q=-\frac{\sqrt{6 c} p_{a t m} l}{16 \rho c h|c-V|^{3 / 2}}$.

First, we consider the case of $c>V$. The phase pattern for that case is shown in Fig. 3 for $Q>0$ (negative atmospheric disturbance). The main regime here is the regime of the trajectory passage corresponding to fast motion of solitons through the source region. The soliton amplitude grows at the moment of interaction (and decreases at the opposite sign of forcing) and recovers after interaction. At the phase plane one can also see (in the region of low amplitudes) trajectories that correspond to generation of virtual solitons. These solitons are generated behind the source, then they grow, take 


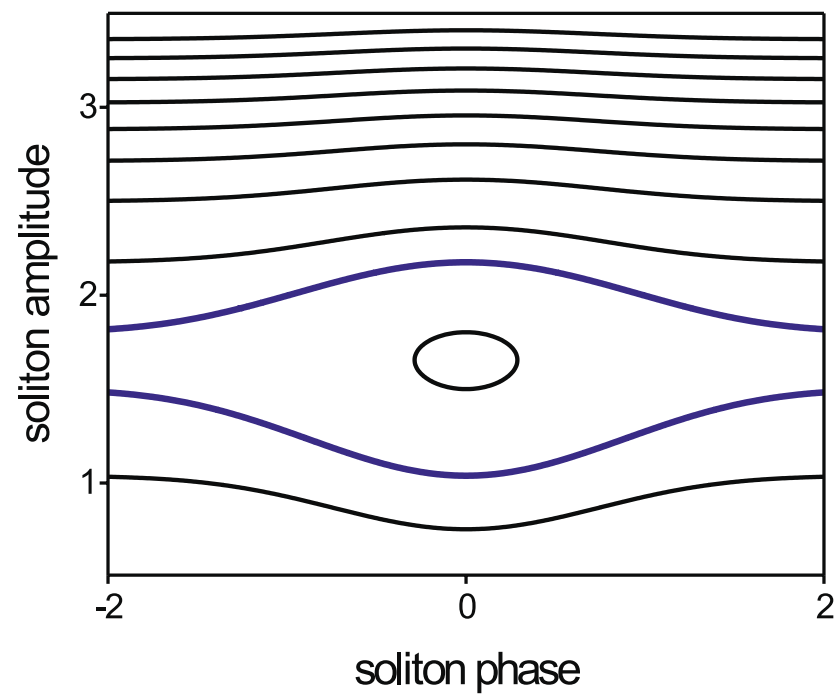

Fig. 4. Phase plane of system (36) and (37) for the "fast" and positive source.

over the source and dissipate. The same regime exists for $Q<0$ (positive atmospheric disturbance); in that case the solitons situated behind the source dissipate during interaction, and a part of the solitons are generated in front of the perturbation.

Let us consider now the opposite case, $c<V$. Dynamics of solitary waves is much richer here. First, the equilibrium state exists

$\alpha a_{0}=3(V-c), \quad \theta=0$.

It is equivalent to the existence of the steady-state soliton propagating with the same velocity as the atmospheric disturbance (resonant generated soliton). Naturally, this is possible only at the certain amplitudes of the solitary wave. The character of the equilibrium depends on the sign of $Q$, in particular, for $Q>0$, it is the center. The phase plane for this case is displayed in Fig. 4. Near the equilibrium point the soliton amplitude and phase oscillate, and this regime corresponds to the soliton capture by the moving source. Here there are also regimes of the trajectory passage and virtual solitary waves, and they are clearly visible on the phase plane.

For $Q<0$ the equilibrium point is the saddle and the corresponding phase plane is displayed in Fig. 5. The main regime here is the reflection of the solitary wave from the moved zone of the atmospheric disturbance. In process of the interaction with the moving forcing, the wave amplitude varies having the next asymptotic values at $\theta \rightarrow \pm \infty$,

$G_{m}\left(1-G_{m}^{2} / 3\right)=G_{M}\left(1-G_{M}^{2} / 3\right)$,

where $m$ and $M$ characterize the minimal and maximal amplitudes. Maximally, wave amplitude $\left(\mathrm{a} \sim \mathrm{G}^{2}\right)$ can change three times in the process of interaction with the moving forcing.

As numerical solutions of the forced Korteweg-de Vries equation showed, the simplified model given here yields a

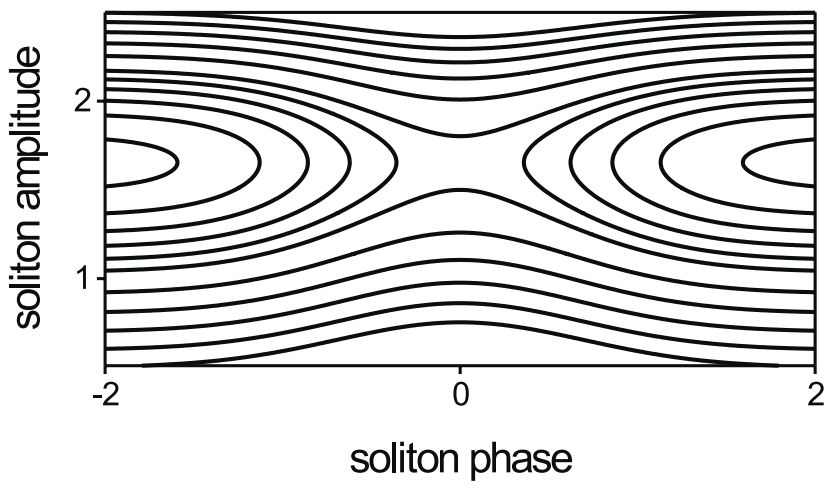

Fig. 5. Phase plane of system (36) and (37) for the "fast" and negative source.

correct physical representation of the interaction between the soliton and the moving forcing (Grimshaw et al., 1994). The generalization of this theory for a case of the forcing moving with variable speed, and also, taking into account the wave dissipation, can be found in papers by Grimshaw et al. (1996, 1997).

The simplified theory given above takes into account the interaction between the solitary wave and the moving forcing. In reality, the number of waves interacting with the atmospheric disturbance can be high and they may intersect and interact between them, sometimes forming largeamplitude waves.

\section{Focusing of nonlinear-dispersive tsunami waves}

Due to nonlinearity and dispersion, the individual waves and wave packets propagate with different velocities and may form a very complicated wave pattern containing the largeamplitude and short-lived impulses. Such impulses in the wind wave field are called the freak or rogue waves, and they are usually studied for deep water (Osborne et al., 2000; Kharif et al., 2001). Some observed unconfirmed data of joint observations of freak and tsunami waves and the link between tsunami and freak waves are discussed by Pelinovsky and Talipova (2001). The effects liked freak waves, in our opinion, are possible for tsunami waves described by the forced Korteweg-de Vries Eq. (30). Let us assume that the moving (in general, with variable speed) zone of the atmospheric disturbances generates the tsunami waves with different characteristics (amplitudes, wavelengths, waveforms). After leaving the generated area, the waves propagate as free waves. In this case Eq. (30) can be reduced to the Korteweg-de Vries equation

$$
\frac{\partial \eta}{\partial t}+c \frac{\partial \eta}{\partial x}+\alpha \eta \frac{\partial \eta}{\partial x}+\beta \frac{\partial^{3} \eta}{\partial x^{3}}=0
$$

The forcing forms the initial conditions for this equation. To demonstrate the wave focusing effect, let us consider the 
linear variant of Eq. (44),

$\frac{\partial \eta}{\partial t}+c \frac{\partial \eta}{\partial x}+\beta \frac{\partial^{3} \eta}{\partial x^{3}}=0$

The general solution of Eq. (45) can be expressed in the Fourier form

$\eta(x, t)=\int \eta(k) \exp [i(\omega t-k x)] d k$,

where

$\eta(k)=\frac{1}{2 \pi} \int \eta(x, 0) \exp i k x d x$

and $\eta(x, 0)$ is the initial waveform. In particular, when the initial condition has the form of a delta-function,

$\eta(x, 0)=S \delta(x)$

with positive (or negative) constant $S$, the integral Eq. (46) is the Airy function (Abramowitz and Stegun, 1964)

$\eta(x, t)=S\left(\frac{2}{c t h^{2}}\right)^{1 / 3} A i\left[\left(\frac{2}{c t h^{2}}\right)^{1 / 3}(x-c t)\right]$,

and the wave field represents the frequency modulated wave train with the leading long wave, its amplitude is decreased, and length is increased. This solution is very often used to demonstrate the dispersion effect, resulting in the wave spreading and attenuation occuring with time. But due to the invariance of Eq. (45) under a transformation $x \rightarrow-x, t \rightarrow$ $-t$, the "inverted" wave packet (Eq. 49) for any fixed moment $T$ will transform into the delta-function (for time, $T$ ) and then it will become dispersive according to Eq. (49). So, the dispersion can lead to the focusing of the wave energy on the finite distances. Of course, the "unbounded" focus is outside of the applicability of the linear long-wave theory. More realistic results can be obtained for the Gaussian impulse with amplitude $A_{0}$ and width $K^{-1}$,

$\eta(x, 0)=A_{0} \exp \left(-K^{2} x^{2}\right)$.

The integral (Eq. 46) is calculated exactly in the explicit form, and the wave field for any time is

$$
\begin{aligned}
& \eta(x, t)=\frac{A_{0}}{K \sqrt[3]{\frac{c t h^{2}}{2}}} \exp \left\{\frac{1}{2 c t h^{2} K^{2}}\right. \\
& \left.\left(x-c t+\frac{6}{77 c t h^{2} K^{4}}\right)\right\} \times A i\left\{\frac{x-c t+\frac{9}{77 c t h^{2} K^{4}}}{\sqrt[3]{\frac{c t h^{2}}{2}}}\right\} .
\end{aligned}
$$

It describes the spreading of the initial Gaussian impulse into the frequency modulated wave packet. The inverted wave packet transformation is described by

$$
\begin{aligned}
& \eta(x, t)=\frac{A_{0}}{K \sqrt[3]{\frac{c(T-t) h^{2}}{2}}} \exp \\
& \left\{\frac{1}{2 c(T-t) h^{2} K^{2}}\left(-(x-c t)+\frac{6}{77 c(T-t) h^{2} K^{4}}\right)\right\} \\
& \times A i\left\{\frac{-(x-c t)+\frac{9}{77 c(T-t) h^{2} K^{4}}}{\sqrt[3]{\frac{c(T-t) h^{2}}{2}}}\right\} .
\end{aligned}
$$
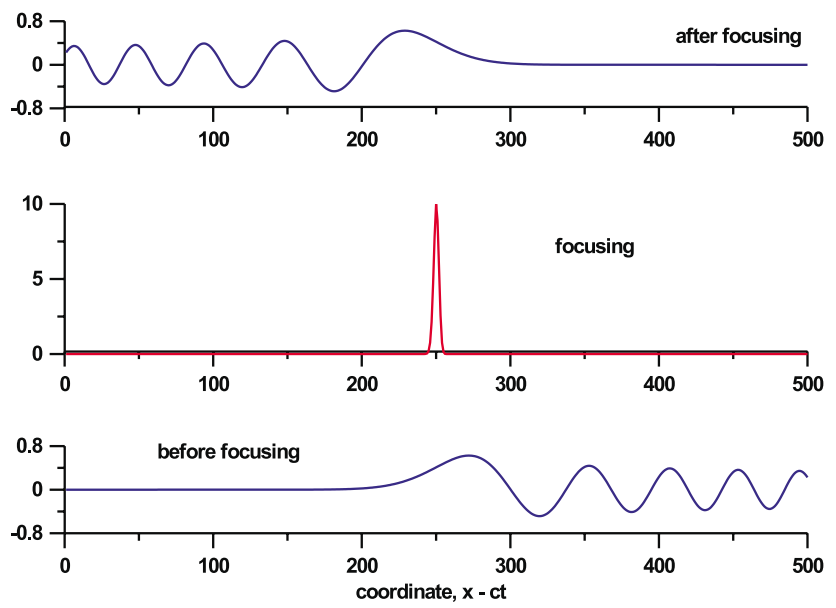

Fig. 6. Focusing of frequency modulated wave packet.

As a result, the frequency modulated wave packet (Eq. 52) transforms into the Gaussian isolated impulse (Eq. 50) for $t \rightarrow T$, and then transforms again into the frequency modulated wave packet (Eq. 51) by replacing $t$ on $t-T$. This process is illustrated in Fig. 6. The amplification in the focal point can be significant. It is very important to note that the appearance of the large-amplitude waves due to a focusing mechanism is not related to the bathymetry features (caustics), and may be on any distance from the shore. This mechanism requires the specific wave generation (weak-speed waves should be generated before strong-speed waves) and this is possible if the meteo-conditions above the sea are enough complicated.

The wave focusing is also possible for the nonlineardispersive waves. The results of the numerical simulation of this process in the framework of the Korteweg-de Vries equation are presented by Pelinovsky et al. (2000).

\section{Conclusion}

The main goal of this paper is to demonstrate possible analytical tests characterizing the role of nonlinearity, dispersion and forcing in the processes of the tsunami generation by atmospheric disturbances; such solutions are important for the interpretation of field data and the results of the numerical simulations. The simplified linear and nonlinear shallowwater models are presented, and their analytical solutions for a basin of constant depth are discussed. The shallow-water model describes well the properties of the generated tsunami waves for all regimes except the resonance case, when the wave amplitude tends to infinity. The nonlinear-dispersive model based on the forced Korteweg-de Vries equation is developed to describe the resonant mechanism of the tsunami wave generation by atmospheric disturbances moving with near-critical speed (long wave speed). Some analytical solutions of the nonlinear dispersive model describing the process of the solitary wave interaction with the moving force are obtained. They illustrate the steady-state and unsteady 
regimes of resonant soliton generation. The focusing of waves in the process of their propagation is investigated. Exact solution of the linear version of the Korteweg-de Vries equation is obtained. Details of the focusing of the frequency modulated wave packets are discussed.

Acknowledgements. This work was supported by the grants from INTAS (99-1637, 99-1068) and RFBR (02-05-65107, 00-0564922).

\section{References}

Abramowitz, M. and Stegun, I. A.: Handbook of mathematical functions. National Bureau of Standards, N. Y., 1964.

Akylas, T. R.: On excitation of long nonlinear water waves by a moving pressure distribution, J. Fluid Mech., 141, 455-466, 1984.

Efimov, V. V., Kulikov, E. A., Rabinovich, A. B., and Fine, I. V.: Waves in the marginal areas of the ocean, Leningrad: Gidrometeoizdat, 1985.

Grimshaw, R., Pelinovsky, E., and Tian, X.: Interaction of Solitary Wave with an External Force, Physica D, 77, 405-433, 1994.

Grimshaw, R., Pelinovsky, E., and Sakov, P.: Interaction of a solitary wave with an external force moving with variable speed, Stud. Applied Mathematics, 97, 235-276, 1996.

Grimshaw, R., Pelinovsky, E., and Bezen, A.: Hysteresis phenomena in the interaction of a damped solitary wave with an external force, Wave Motion, 26, 253-274, 1997.

Kajiura, K.: The leading wave of a tsunami., Bull. Earthq. Res. Inst., 41, 535-571, 1963.

Kharif, C., Pelinovsky, E., Talipova, T., and Slunyaev, A.: Focusing of nonlinear wave groups in deep water, JETP Letters, 73, 4, 170-175, 2001.

Marchuk, A. G., Chubarov, L. B., and Shokin, Yu. I.: Numerical modelling of tsunami waves, Novosibirsk, Nauka, 1983.

Murty, T.: Seismic sea waves - Tsunamis, Bull. Fish. Res. Boand Canada, Ottawa, 198, 1977.

Nosov, M. A. and Shelkovnikov, N. K.: Tsunami generation by moving bottom displacement, Vestnik of Moscow University,
Ser. 3., 36, 96-101, 1995.

Novikova, L. E. and Ostrovsky, L. A.: Excitation of tsunami waves by a travelling displacement of the ocean bottom, Marine Geodesy, 2, 365-380, 1978.

Osborne, A. R., Onorato, M., and Serio, M.: The nonlinear dynamics of rogue waves and holes in deep-water gravity wave train, Phys. Letts. A, 275, 386-393, 2000.

Pelinovsky, E. N.: Nonlinear dynamics of tsunami waves, Institute of Applied Physics, Gorky, 1982.

Pelinovsky, E. N.: Hydrodynamics of tsunami waves, Institute of Applied Physics, Nizhny Novgorod, 1996.

Pelinovsky, E. N. and Choi, H. S.: A Mathematical Model for Nonlinear Waves due to Moving Disturbances in a Basin of Variable Depth, J. Korean Soc. Coastal and Ocean Eng., 5, 191-197, 1993.

Pelinovsky, E. N. and Poplavsky, A.: Simplified model of tsunami generation by submarine landslides, Phys. Chem. Earth, 21, 1317, 1996.

Pelinovsky, E. N. and Talipova, T.: Does the link between freak and tsunami wave exist? Abstracts of the NATO Advanced Research Workshop "Underwater Failure Mechanisms on Tsunami Generation, Modeling, Tsunami Risk and Mitigation" (Istanbul, Turkey, May 2001), 168-172, 2001.

Pelinovsky, E. N., Talipova, T., and Kharif, C.: Nonlinear dispersive mechanism of the freak wave formation in shallow water, Physica D, 147, 83-94, 2000.

Rabinovich, A. B.: Long gravity waves in the ocean: capture, resonance, leaking, St. Petersburg, Gidrometeoizdat, 1993.

Rabinovich, A. and Monserrat, S.: Generation of meteorological tsunamis (large amplitude seiches) near the Baleric and Kuril Islands, Natural Hazards, 18, 27-55, 1998.

Tinti, S. and Bortolucci, E.: Energy of water waves induced by submarine landslides, Pageoph, 157, 281-318, 2000a.

Tinti, S. and Bortolucci, E.: Analytical investigation of tsunamis generated by submarine slides, Annali di Geofisica, 43, 519-536, $2000 b$.

Tinti, S., Bortolucci, E., and Chiavettieri, C.: Tsunami excitation by submarine slides in shallow-water approximation, Pageoph, 158, 759-797, 2001. 